\title{
Investigation of Drain Disturb in SONOS Flash EEPROMs
}

\author{
P. Bharath Kumar, Student Member, IEEE, Ravinder Sharma, Pradeep R. Nair, and \\ Souvik Mahapatra, Member, IEEE
}

\begin{abstract}
The mechanism of drain disturb is studied in siliconoxide-nitride-oxide-silicon Flash electrically erasable programmable read-only memory cells. It is shown that disturb is a serious problem in programmed cells and is caused by injection of hot holes from substrate into the oxide/nitride/oxide stack. The origin of these holes is identified by analyzing the influence of halo doping, channel doping, and channel length scaling on drain disturb. Band-to-band tunneling at the drain junction is normally the dominant source of these holes. It is also shown that holes generated out of impact ionization of channel electrons become dominant in cells with high channel leakage (especially at lower channel lengths). Finally, the effect of repeated program/ erase cycling on drain disturb is studied. Drain disturb becomes less severe with cycling, the reasons for which are determined using gate-induced drain leakage measurements and device simulations.
\end{abstract}

Index Terms-Band-to-band tunneling (BTBT), charge pumping $(\mathrm{CP})$, drain disturb, Flash electrically erasable programmable read-only memories (EEPROMs), gate-induced drain leakage (GIDL), hot holes, silicon-oxide-nitride-oxide-silicon (SONOS).

\section{INTRODUCTION}

$\mathbf{F}$ LOATING-GATE (FG) Flash electrically erasable programmable read-only memory (EEPROM), which has been the primary nonvolatile semiconductor memory for a long time is stated to reach its scaling limitations in a few years [1]. Among the various alternative nonvolatile memories that are being explored, silicon-oxide-nitride-oxide-silicon (SONOS)type (e.g., NROM) nitride-based charge-trapping memories come closest to replacing FG EEPROMs in the future generations [1]-[4]. In addition to being more scalable than FG EEPROMs, they require fewer masking steps to integrate with core logic, thereby making them attractive for embedded applications as well [2], [3]. When operated using localized charge injection mechanisms (NOR applications) for program and erase, they provide low-voltage operation and also offer multibit-per-cell storage capability through spatial separation of trapped charges [2]-[4]. However, SONOS Flash cells operated

Manuscript received April 13, 2006; revised August 8, 2006. The review of this paper was arranged by Editor S. Kimura.

P. B. Kumar and S. Mahapatra are with the Department of Electrical Engineering, Indian Institute of Technology Bombay, Mumbai 400076, India (e-mail: bharath@ee.iitb.ac.in).

R. Sharma was with the Department of Electrical Engineering, Indian Institute of Technology Bombay, Mumbai 400076, India. He is now with the STMicroelectronics Pvt., Ltd., Noida 201301, India.

P. R. Nair was with the Department of Electrical Engineering, Indian Institute of Technology Bombay, Mumbai 400076, India. He is now with the School of Electrical and Computer Engineering, Purdue University, West Lafayette, IN 47907-1285 USA.

Digital Object Identifier 10.1109/TED.2006.887232 under localized charge injection suffer from program/erase (P/E) cycling endurance and data retention problems, and much work has been done in the past to understand and improve these reliability issues [4]-[7]. This paper shows that in addition to endurance and retention, drain disturb can also become a serious issue for such modes of operation and must be considered for reliable design of memory cells. The mechanism behind drain disturb is established and its dependence on cell doping, program bias, and P/E cycling is discussed. This is an enhanced version of our earlier work [8] and the study of drain disturb in $\mathrm{P} / \mathrm{E}$-cycled cells is presented in addition.

Drain disturb or drain stress occurs in gate unselected cells of a NOR Flash array that share the bit line of a cell being programmed using hot-carrier injection [9]. FG EEPROMs suffer from disturb in both programmed and erased states due to charge loss and charge gain, respectively [10]. But drain disturb in SONOS cells is found to be serious only in programmed state, which leads to a continuous drop in threshold voltage $\left(V_{\mathrm{TH}}\right)$. Although the result is similar to retention loss caused by redistribution and decay of charges in the topoxide/nitride/bottom-oxide (ONO) stack [5]-[7], [11], drain disturb is found to involve hole injection from $\mathrm{Si}$ substrate into the ONO stack. The sources of these holes are determined by studying the effect of channel length, channel doping and bias on disturb. P/E cycling is seen to reduce drain disturb, the reasons for which are also explored.

The details of the devices, measurements, and simulations used in this paper are presented in Section II. Section III-A discusses the effect of drain disturb on the cell $V_{\mathrm{TH}}$ and its dependence on drain bias. The mechanism behind drain disturb is established in Section III-B by studying the effect of channel length, doping, and substrate bias on disturb. The effect of $\mathrm{P} / \mathrm{E}$ cycling on disturb is discussed in Section III-C. Conclusions are presented in Section IV.

\section{Device, Measurement, and Simulation Details}

Measurements were performed on isolated stack-gate SONOS cells with ONO thickness of $5.8 / 8 / 5 \mathrm{~nm}$, width of $2 \mu \mathrm{m}$, and drawn gate lengths $(L)$ of 0.25 and $0.35 \mu \mathrm{m}$. To study the effect of doping on drain disturb, cells with additional implants (halo and channel compensation) were used. Fig. 1 shows the schematic of the cell along with different doping implants. Channel compensation implants used in this paper reduce the natural $V_{\mathrm{TH}}$ of the cell, and as well reduce the programming speed under channel-hot-electron (CHE) injection (due to lower fields at drain junction). Halo implants increase 


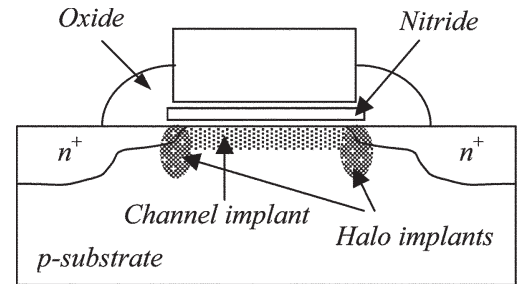

Fig. 1. Schematic of SONOS cells used in this paper, showing different implants (details in Table I). The ONO dimensions are 5.8/8/5 nm and the gate stack length is $250 \mathrm{~nm}$ (unless mentioned otherwise).

TABLE I

Doping Details of THE SONOS CELLS USED FOR MEASUREMENTS Along With Typical Natural $V_{\text {Th }}$ Values and Program Times. Program Time $\left(T_{P}\right)$ Was Measured for a Constant $V_{\text {Th }}$ Shift $(\sim 2.5 \mathrm{~V})$ From VIRGIN $V_{\mathrm{TH}}$, AT A FIXEd PROGRAM BIAs $8 / 5 / 0 \mathrm{~V}$

\begin{tabular}{|c|c|c|c|c|}
\hline Device & $\begin{array}{c}\text { Channel } \\
\text { Implant }\end{array}$ & $\begin{array}{c}\text { Halo } \\
\text { Implant }\end{array}$ & $\begin{array}{c}\text { Natural } \\
\mathbf{V}_{\text {TH }}(\mathbf{V})\end{array}$ & $\begin{array}{c}\text { Typical } \\
\mathbf{T} \mathbf{P}(\boldsymbol{\mu s})\end{array}$ \\
\hline Normal & $\mathrm{B}$ & - & 2.5 & $\sim 20$ \\
\hline Compensated & $\begin{array}{c}\mathrm{B}, \mathrm{BF}_{2}, \\
\mathrm{As}\end{array}$ & - & 0.9 & $\sim 100$ \\
\hline Halo & $\mathrm{B}$ & $\mathrm{B}$ & 3.2 & $\sim 7$ \\
\hline
\end{tabular}

the programming speed under CHE injection (higher fields at drain junction) as well as the natural $V_{\mathrm{TH}}$ of the cell. Table I lists the different implants used, along with corresponding $V_{\mathrm{TH}}$ values. Most of the experiments were done on normal cells (no compensation, no halo) unless mentioned otherwise.

Programming was done using CHE injection at a bias $\left(V_{G} / V_{D} / V_{B}\right)$ of $8 / 5 / 0 \mathrm{~V}$. Erase was done through injection of hot holes at a bias of $-8 / 5 / 0$, unless mentioned otherwise. These holes are generated by band-to-band tunneling (BTBT) in the drain region and are heated by the lateral field in drain-substrate junction before injection [12]. Drain disturb measurements were done by grounding the gate terminal and maintaining other terminals at same bias as during program. $I_{D}-V_{G}$ measurements were taken in reverse read mode by interchanging source and drain terminals during read [2]. $V_{\mathrm{TH}}$ was determined from $I_{D}-V_{G}$ using constant current definition $\left(I_{D}=8 \mu \mathrm{A}\right.$ at $\left.V_{D}=0.1 \mathrm{~V}\right)$. Charge-pumping $(\mathrm{CP})$ measurements were done using fixed base $\left(V_{\mathrm{BASE}}=-4.6 \mathrm{~V}\right)$ and varying top $\left(V_{\text {TOP }}\right.$ up to $\left.+4.6 \mathrm{~V}\right)$ gate pulse having frequency of $400 \mathrm{kHz}$. Gate-induced drain leakage (GIDL) measurements [13] were done at $V_{D}$ of $2 \mathrm{~V}$, which is high enough to give reasonable $I_{\mathrm{GIDL}}$ but low enough not to disturb the cell $V_{\mathrm{TH}}$. $I_{D}-V_{G}$ measurements were taken at regular time intervals during program, erase and disturb, while CP and GIDL measurements were done at the start and end of program, erase and disturb.

Well-calibrated ISE-TCAD tools DIOS and DESSIS [14] were used for process and device simulations, respectively. A nonself-consistent full-band Monte Carlo simulator [15] was used for simulating hot carrier distributions in silicon. The phonon and impact ionization scattering parameters were adjusted to obtain good match with experimental and simulated substrate and gate currents. The device potentials used in Monte Carlo simulations were obtained from DESSIS.

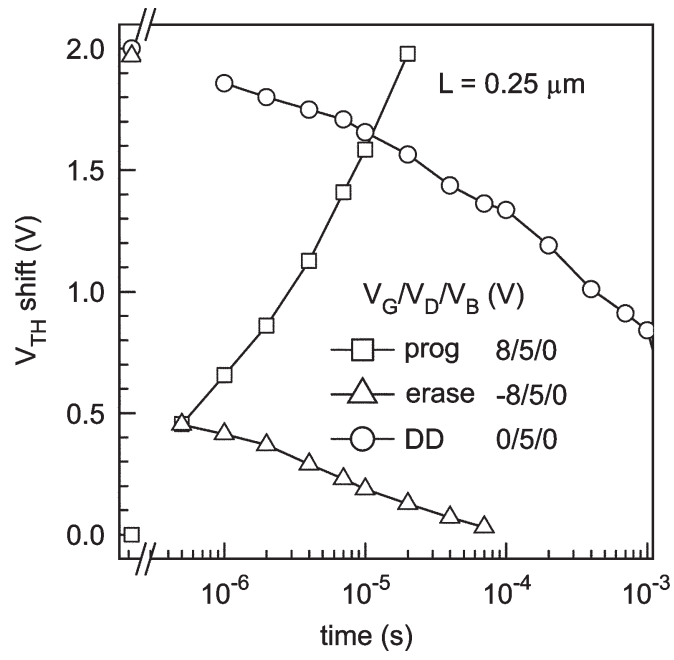

Fig. 2. Measured $V_{\mathrm{TH}}$ shift (with respect to virgin $V_{\mathrm{TH}}$ ) in a SONOS cell during program, erase and program state drain disturb conditions. No drain disturb was observed in erase state in the time scale shown here. Erase and disturb measurements were done on two different cells programmed to same program $V_{\mathrm{TH}}$.

\section{RESUlTS AND DiscUSSION}

\section{A. Identification of Disturb Mechanism}

Fig. 2 shows the experimental program, erase and drain disturb (referred to as disturb from now) transients of a SONOS cell. The erased $V_{\mathrm{TH}}$ is the same as the virgin cell $V_{\mathrm{TH}}$ and programmed state was fixed at a $V_{\mathrm{TH}}$ of $2 \mathrm{~V}$ above virgin $V_{\mathrm{TH}}$. A huge drop of $V_{\mathrm{TH}}$ is observed under disturb condition in a programmed cell, while erased cell does not show any significant change of $V_{\mathrm{TH}}$ in the same time scale (not shown). This clearly shows that drain disturb can be a serious problem in programmed cells. In a localized CHE programmed SONOS cell, electrons trapped near the drain side of ONO stack are responsible for increasing the $V_{\mathrm{TH}}$ (local high $V_{\mathrm{TH}}$ determines the overall cell $\left.V_{\mathrm{TH}}\right)$. Therefore, the decrease in $V_{\mathrm{TH}}$ during disturb can occur due to redistribution of these trapped electrons in ONO, their escape from ONO (similar to that of retention loss [11]), or injection of holes into the ONO stack (similar to that of erase) and needs to be resolved.

Fig. 3 shows measured $V_{\mathrm{TH}}$ drop during disturb as a function of drain bias. $V_{\mathrm{TH}}$ drop increases at higher $V_{D}$ as shown and this drop is monotonous with time for all $V_{D}$. These observations rule out vertical or lateral charge redistribution in ONO to be the major cause behind disturb, as explained below. In these programmed cells, the lateral spread of trapped electrons was found to be $40-50 \mathrm{~nm}$, of which about $30-40 \mathrm{~nm}$ is above channel region and the rest in gate-drain overlap region [16]. The peak of this distribution is near the gate edge and the magnitude gradually decreases toward center of the channel. The cell $V_{\mathrm{TH}}$ is mainly determined by electrons trapped above the channel region. Vertical movement of these electrons away from the $\mathrm{Si} / \mathrm{SiO}_{2}$ interface or a decrease in their peak concentration due to their lateral redistribution (toward center of the channel) can reduce $V_{\mathrm{TH}}$. Increasing $V_{D}$ is unlikely to accelerate movement of electrons in either of these modes that result in a monotonous $V_{\mathrm{TH}}$ drop. Hence, redistribution of trapped electrons in $\mathrm{ONO}$ cannot be the main cause behind drain disturb. 


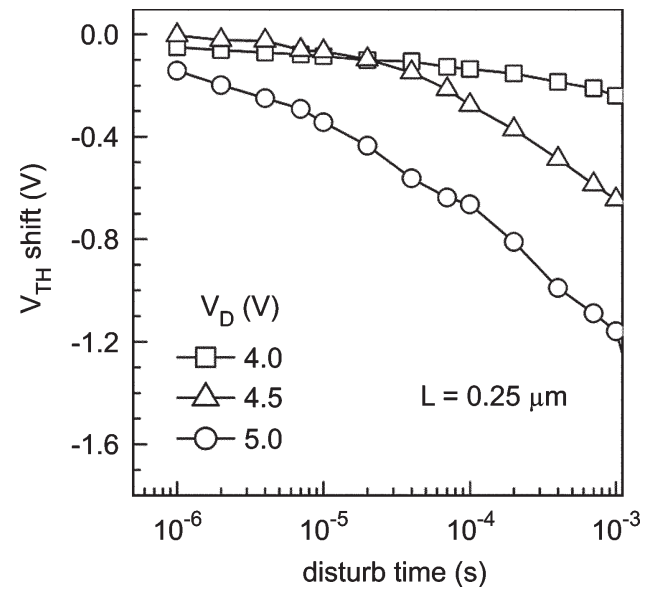

Fig. 3. Measured drain disturb transients of a programmed SONOS cell at different disturb $V_{D}$. Drain disturb was measured on different cells programmed to the same level and the $V_{\mathrm{TH}}$ shift shown is with respect to the programstate $V_{\mathrm{TH}}$.

Under disturb condition, a programmed cell is in OFF state and changing $V_{D}$ mainly affects the fields in drain-substrate junction and gate-drain overlap region. Therefore, disturb mechanism should be accelerated by fields in these regions, which makes hot-hole injection into the ONO stack a likely cause for disturb. Due to absence of drain coupling in SONOS cells, $V_{D}$ does not have much control over vertical fields in ONO above the channel region. Furthermore, electron ejection from drain-gate overlap region cannot lead to significant $V_{\mathrm{TH}}$ shifts. The mechanism of hot-hole generation under disturb condition could be similar to that in erase, except for a lower vertical field in drain-gate overlap region as the gate is unselected. This is explored next by comparing disturb with erase.

To compare disturb and erase, the distributions of hot holes generated by BTBT under these conditions were simulated using a Monte Carlo simulator. A programmed cell with charge (equivalent to the ONO trapped charge) placed at the $\mathrm{Si} / \mathrm{SiO}_{2}$ interface was used for these simulations [16]. Fig. 4 compares the lateral profiles of channel hot-hole density and transverse electric field along channel in a programmed SONOS cell under erase and disturb conditions. The profiles of hot-hole distribution and vertical field during disturb are similar to erase, although with lower magnitudes, suggesting injection of BTBT generated holes as a possible cause for disturb.

CP [16]-[18] is used to verify hole injection and any resultant generation of interface traps $\left(N_{\mathrm{IT}}\right)$ after disturb and erase. Fig. 5 compares $\mathrm{CP}$ current $\left(I_{\mathrm{CP}}\right)$ measured using fixed base and varying top method in SONOS cells after erase and disturb. For this, two identically programmed cells were separately subjected to erase and disturb (a longer disturb time was used to get similar $V_{\mathrm{TH}}$ shift as in erase). $I_{\mathrm{CP}}$ before and after programming (similar for both these cells) is also shown for comparison. A large increase in $I_{\mathrm{CP}}$ (due to $N_{\mathrm{IT}}$ generation) is seen after erasing the cell. It is well known that hole injection in MOSFET causes $N_{\text {IT }}$ generation [19]-[21] and the increased $I_{\mathrm{CP}}$ after erase reflects this. A similar large $I_{\mathrm{CP}}$ increase is also seen after drain disturb (longer time) suggesting hole injection during disturb too. Moreover, note that the rising edge of $I_{\mathrm{CP}}-V_{\mathrm{TOP}}$ curve shifts to lower $V_{\mathrm{TOP}}$ value after disturb

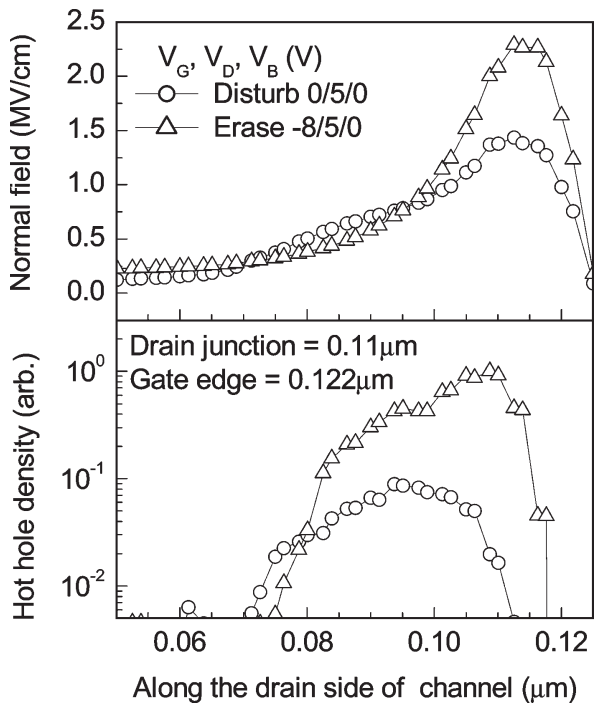

Fig. 4. Simulated profiles of normal electric field and channel hot-hole concentration (Monte Carlo simulated) across the channel in a programmed SONOS cell under erase and drain disturb conditions. To simulate the program state, charge equivalent to the program trapped charge is placed at $\mathrm{Si} / \mathrm{SiO}_{2}$ interface [16].

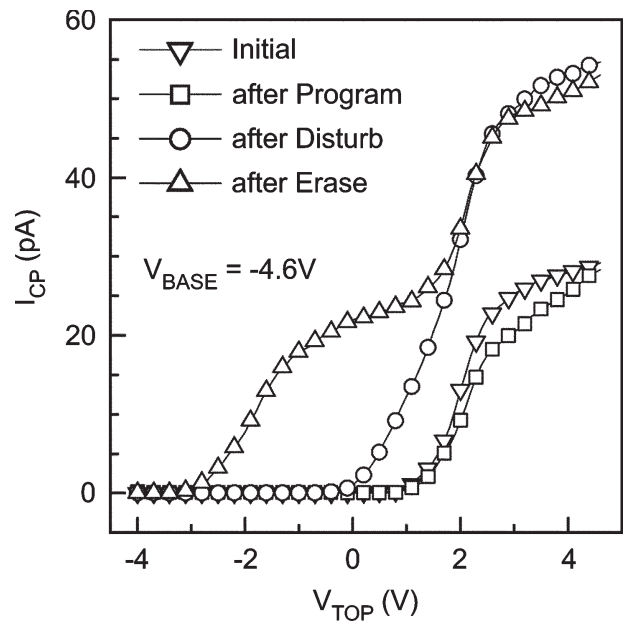

Fig. 5. $I_{\mathrm{CP}}$ of SONOS cells measured using a gate pulse with a fixed base and varying top. $I_{\mathrm{CP}}$ characteristics after drain disturb and erase (in two different cells programmed to same $V_{\mathrm{TH}}$ level) are shown along with virgin and programmed cell characteristics. A longer disturb time was used to get similar $V_{\mathrm{TH}}$ shift as in erase.

and erase compared to the virgin/programmed cells. This is due to reduced local $V_{\mathrm{TH}}$ after erase/disturb as compared to virgin/programmed cells. It can be reasonably assumed that erase and disturb do not significantly alter $V_{\mathrm{TH}}$ and $V_{\mathrm{FB}}$ profiles near the source and center of channel. The reduction in $V_{\mathrm{TH}}$, therefore, occurs near the drain region and indicates hole injection in that region. Loss of electrons (those injected during program) cannot be the dominant cause for this shift in $I_{\mathrm{CP}}-V_{\mathrm{TOP}}$ curve as that would never shift the rising edge of postdisturb or posterase $I_{\mathrm{CP}}$ curve beyond the virgin curve. The much larger shift of the rising edge after erase compared to that after disturb indicates a larger reduction in the local $V_{\mathrm{TH}}$ after erase due differences in injected hot-hole profiles. 


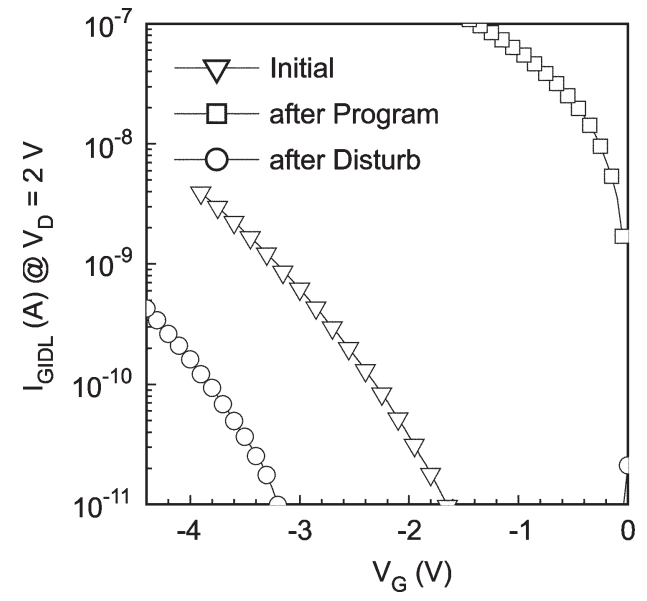

Fig. 6. GIDL currents in a SONOS cell measured before and after drain disturb in programmed state. The GIDL current before programming (initial) is also shown for comparison. GIDL curve gradually shifts to the left during disturb and even shifts beyond the initial (preprogram) curve for sufficiently large disturb like the one shown here.

As a further confirmation for hole injection during disturb, Fig. 6 compares the measured GIDL characteristics before and after disturb in a programmed cell. Unlike $V_{\mathrm{TH}}$, GIDL current $\left(I_{\mathrm{GIDL}}\right)$ is very sensitive to the charges in gate-drain overlap region, as it is exponentially dependent on vertical field in that region [22]. GIDL curve shifts toward more positive $V_{G}$ (larger $I_{\mathrm{GIDL}}$ at a given $V_{G}$ ) after programming due to electron injection into the ONO above overlap. During disturb, GIDL curve gradually shifts back toward more negative $V_{G}$ indicating a decrease in negative charges in the overlap region. However, the shift of postdisturb (after sufficiently long disturb time) GIDL curve beyond the initial curve indicates hole injection during disturb, as only electron ejection would bring the postdisturb GIDL curve close to (not beyond) the initial curve.

\section{B. Impact of Device Structure and Doping}

The above results clearly show that hole injection into ONO stack is the key cause for drain disturb in a programmed SONOS cell. Similar to erase, disturb involves BTBT at the drain junction, heating up of resultant holes in the junction lateral fields, and their subsequent injection into the ONO stack. Under disturb condition, trapped electrons in ONO together with $V_{D}$ can provide the necessary vertical fields in the gatedrain overlap region for BTBT and hole injection. To verify this further, the dependence of drain disturb on channel length $(L)$, channel doping, and halo implants is studied next. BTBT is independent of $L$, but depends on both channel doping and halo implants, which change the fields in the drain-substrate junction. The fields are lower in the presence of compensation, leading to reduction in hot holes (less heating). On the other hand, halo implants increase these local fields resulting in larger heating.

Fig. 7 shows the $V_{\mathrm{TH}}$ shift as a function of time, measured in cells with and without halo doping (no compensation) under disturb condition at different $V_{D}$. The presence of halo increases disturb as expected (larger field and BTBT), which agrees well with hot-hole injection being the dominant cause

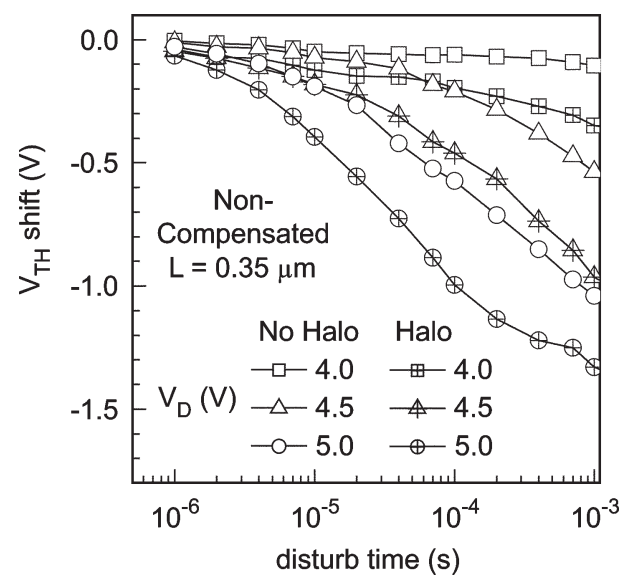

Fig. 7. Drain disturb transients measured at different $V_{D}$ in SONOS cells with and without halo implants. The $V_{\mathrm{TH}}$ shift shown is with respect to the programstate $V_{\mathrm{TH}}$. Before taking the disturb measurements, the cells were programmed for a $V_{\mathrm{TH}}$ shift of $2 \mathrm{~V}$ from virgin state.

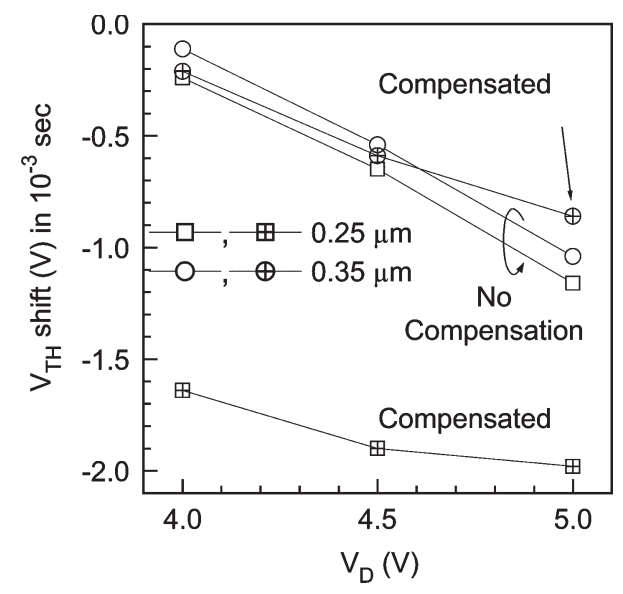

Fig. 8. $\quad V_{\mathrm{TH}}$ shift under drain disturb (for fixed time) in SONOS cells with and without compensation implant at different $L$. The cells were programmed for a $V_{\mathrm{TH}}$ shift of $2 \mathrm{~V}$ from virgin state, before measuring disturb.

of disturb in SONOS cells. Fig. 8 shows the $V_{\mathrm{TH}}$ shift under drain disturb (for fixed time) as a function of $V_{D}$ in SONOS cells of different $L$, with and without compensation implants. Disturb increases at higher $V_{D}$ for all $L$ and for both normal and compensated cells. Normal cells show weak dependence of disturb on $L$ (for all $V_{D}$ ) as BTBT is weakly dependent on $L$. However, compensated cells show a large increase in disturb as $L$ is reduced, contrary to expectation. For the present case, channel compensation influences disturb only slightly in $L=0.35 \mu \mathrm{m}$ cells, while it drastically increases disturb in $L=0.25 \mu \mathrm{m}$ cells (for all $V_{D}$ ). This suggests the possibility of another cause for drain disturb in compensated cells, which is strongly activated at low $L$.

It is unlikely that the presence of compensation changes the fact that hole injection, and not electron loss or redistribution, is the main cause for disturb. It is more likely that more hot holes are being injected in compensated cells having lower $L$. One possible source of this additional component could be the channel leakage current. Localized charge trapping in SONOS cells degrades the subthreshold slope of programmed cells [16], [22], which can lead to channel leakage current even under disturb 


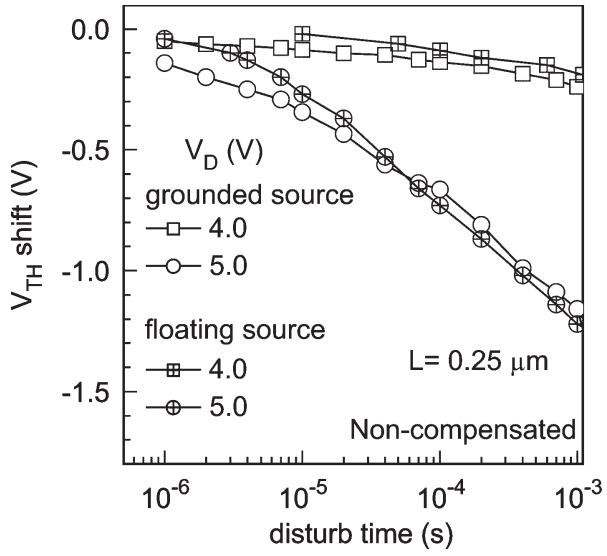

(a)

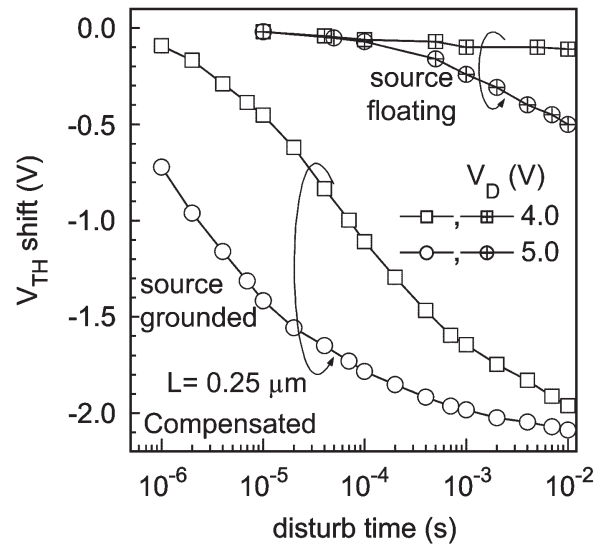

(b)

Fig. 9. Drain disturb transients measured with source terminal grounded and floating, in SONOS cells (a) without and (b) with compensation implant. Floating the source terminal during disturb eliminates the channel leakage current.

conditions, especially at scaled $L$. The channel electrons can undergo impact ionization due to large lateral fields and the generated holes can also get heated and injected into ONO stack. Compensated cells have a lower $V_{\mathrm{TH}}$, resulting in higher channel leakage current under similar disturb bias, which could lead to larger disturb in these cells.

To confirm the presence of channel leakage component in disturb, and compare its contribution with BTBT component, separate disturb measurements were done with the source terminal open. An open source terminal eliminates channel leakage current but does not affect BTBT near drain junction. Fig. 9 shows disturb transients in cells with and without compensation, measured with source terminal grounded and open. As expected, disturb in noncompensated cells changes very little when source terminal is open, indicating that main contribution is from BTBT. On the contrary, cells with compensation show a drastic reduction in disturb when the source terminal is open, indicating that channel leakage is a significant contributor to disturb in these cells. As BTBT and channel leakage show opposite trends with $V_{B}$, studying the dependence of disturb on $V_{B}$ can also confirm the presence of these two components.

Note that as $V_{B}$ becomes more negative, BTBT increases but channel leakage current decreases (due to body effect).

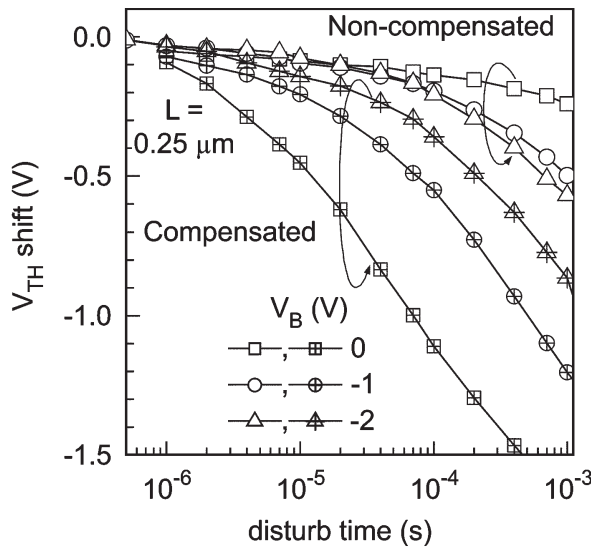

(a)

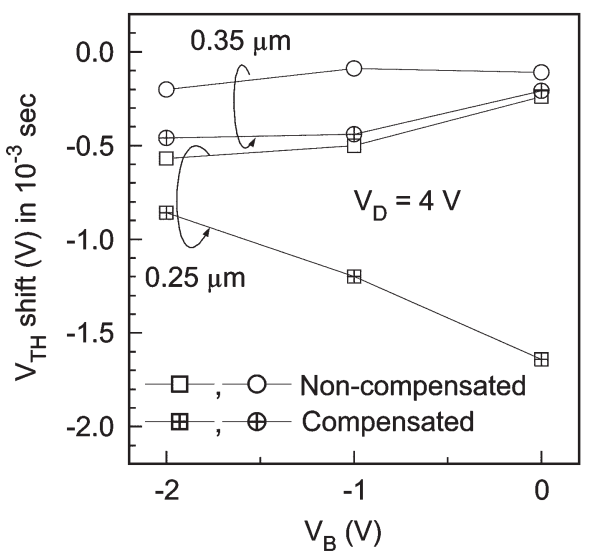

(b)

Fig. 10. (a) Drain disturb transients measured at different $V_{B}$ in programmed SONOS cells with and without compensation implant. Increasing $\left|V_{B}\right|$ increases BTBT but reduces channel leakage. (b) Measured $V_{\mathrm{TH}}$ shift during drain disturb (fixed time) as a function of $V_{B}$ in SONOS cells with and without compensation, at different $L$.

Fig. 10(a) shows disturb transients as a function of $V_{B}$ in normal and compensated cells. A smaller $L$ is deliberately chosen to increase leakage component for compensated cells. Disturb increases with increasing $\left|V_{B}\right|$ in normal cells showing BTBT as the dominant cause for disturb in these cells. Disturb decreases with $\left|V_{B}\right|$ in compensated cells showing channel leakage as the dominant component leading to disturb. However, note that channel leakage would reduce at higher $L$ even for compensated cells and this also should reflect in the disturb dependence on $V_{B}$. Fig. 10(b) shows $V_{\mathrm{TH}}$ shift during disturb as a function of $V_{B}$ in cells with different $L$. Decrease of disturb with increasing $\left|V_{B}\right|$ is observed only in shorter compensated cells but not the longer ones, which confirms channel leakage as the main cause behind increased disturb in compensated cells having scaled $L$.

In summary, the above results clearly show that injection of holes from substrate into ONO stack causes drain disturb in programmed SONOS cells. The main source of these holes is BTBT at the gate-drain overlap. In addition, holes can also get generated from impact ionization initiated by channel leakage current, which can become dominant at lower $L$ in cells with low channel doping. 


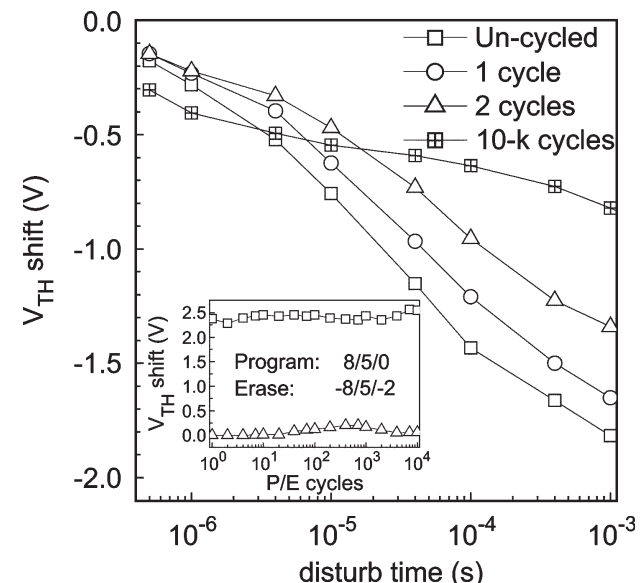

Fig. 11. Drain disturb transients in programmed SONOS cells as a function of P/E cycling. The comparatively larger initial $V_{\mathrm{TH}}$ drop in $10 \mathrm{k}$ cycled cell is due to the degraded retention with cycling [11]. The program and erase biases were chosen for a good endurance. Inset shows the P/E cycling window.

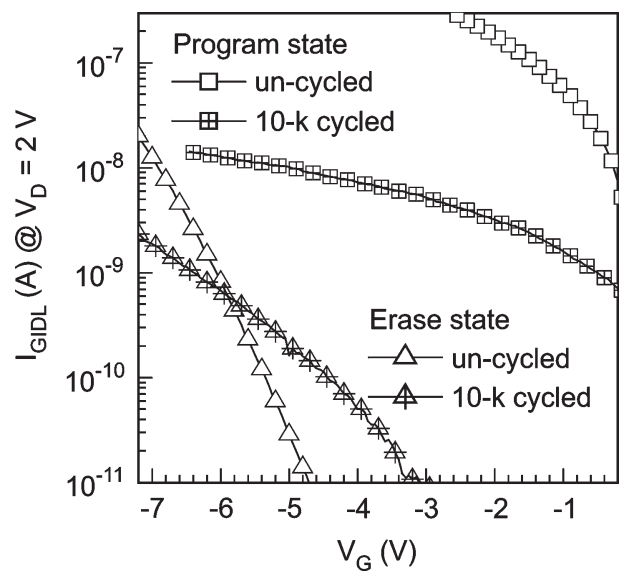

Fig. 12. Program-state and erase-state GIDL currents measured in a SONOS cell before and after 10k cycling. The corresponding P/E cycling window is shown in the inset of Fig. 11.

\section{Effect of P/E Cycling on Drain Disturb}

Fig. 11 shows the effect of P/E cycling on charge loss drain disturb in a programmed normal SONOS cell (inset shows the $V_{\mathrm{TH}}$ window during cycling). Disturb gradually decreases with $\mathrm{P} / \mathrm{E}$ cycling, starting from the first $\mathrm{P} / \mathrm{E}$ cycle. As disturb originates from BTBT at the gate-drain overlap for normal uncompensated cells, Fig. 12 shows GIDL characteristics measured in uncycled and 10k cycled cells. The reduction of programstate $I_{\mathrm{GIDL}}$ after cycling clearly shows a reduction in BTBT, which is consistent with reduction of program-state disturb in cycled cells. GIDL characteristics in the cycled cell also show a distinct skew compared those in the uncycled cell (the difference in $I_{\mathrm{GIDL}}$ in uncycled and cycled cells becomes larger at larger $\left|V_{G}\right|$ values).

Compared to virgin cell, a P/E cycled cell has additional interface traps $\left(I_{\mathrm{CP}}\right.$ continually increases with cycling starting from the first one [11], shown later). Furthermore, repeated $\mathrm{P} / \mathrm{E}$ cycling can also result in accumulation of trapped electrons above the channel region [23]. However, this cannot be significant for the present $\mathrm{P} / \mathrm{E}$ conditions because of the good memory window observed during cycling (shown in the inset of

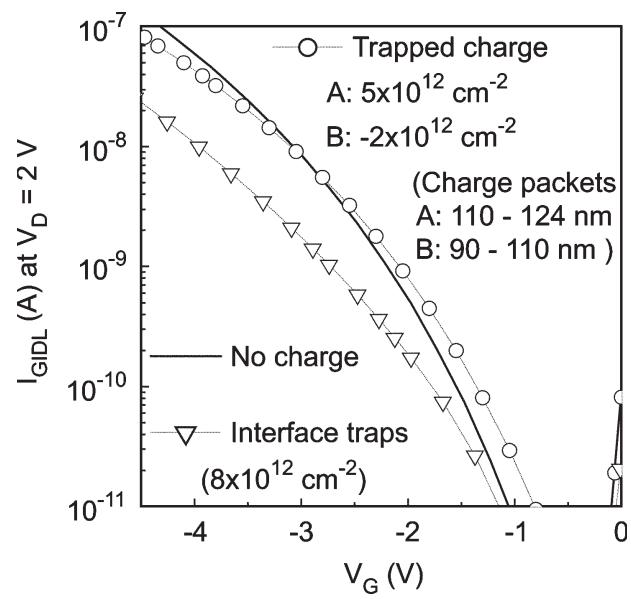

Fig. 13. Simulated GIDL curves of a SONOS cell in the presence of ONO trapped charge and interface traps. Trapped charge is placed at the $\mathrm{Si} / \mathrm{SiO}_{2}$ interface in two Gaussian packets (packet A in overlap and packet B above channel) and interface traps are placed uniformly across the channel (traps are donor type in lower half and acceptor type in the upper half, and are uniformly distributed in energy). The skew in GIDL curve occurs in the presence of either a charge dipole (positive charge in A and negative charge in B), or interface traps.

Fig. 11). Charge accumulation can still be present in the overlap region, which does not affect $V_{\mathrm{TH}}$ window but significantly affects GIDL, which is analyzed below.

Before proceeding further, we wish to point out that a reduction of $I_{\text {GIDL }}$ (which originates from BTBT) also causes erase speed to reduce after cycling (not shown). However, its effect is not seen on the cycling window (inset of Fig. 11) as a slightly longer erase time, than needed to get the required $V_{\mathrm{TH}}$ shift, was used here. During BTBT erase, localized hole injection causes $V_{\mathrm{TH}}$ to saturate at longer erase time and erasing the cell for longer time has very little effect on $V_{\mathrm{TH}}$ transient. Note that when $V_{\mathrm{TH}}$ is laterally nonuniform along the channel, the region with highest local $V_{\mathrm{TH}}$ controls $I_{D}$ and determines the overall cell $V_{\mathrm{TH}}$. A cell programmed using CHE injection has its peak $V_{\mathrm{TH}}$ near drain. Once these injected electrons are erased, further hole injection in that region has no effect on cell $V_{\mathrm{TH}}$ unless the hole injection region is very wide, spreading deep into the channel. So using longer erase time has no effect on erase state $V_{\mathrm{TH}}$ at the start of cycling, but helps to offset any reduction in erase speed with cycling and results in a good cycling window [11].

Simulations were used to qualitatively study the effect of trapped charges and $N_{\mathrm{IT}}$ on GIDL characteristics. Trapped charges were placed at the $\mathrm{Si} / \mathrm{SiO}_{2}$ interface as two Gaussian packets on either side of drain junction-one in overlap (packet A) and one above channel (packet B), and interface traps were placed uniformly across the interface. Fig. 13 illustrates two sample cases where trapped charge and interface traps skew the simulated GIDL curve compared to the curve with no charge or traps. Negative trapped charge in both packets A and B increases $I_{\text {GIDL }}$ for all $V_{G}$ and shifts the GIDL curve toward higher $V_{G}$ (no skew; not shown). Similarly, positive trapped charge in both packets $\mathrm{A}$ and $\mathrm{B}$ decreases $I_{\mathrm{GIDL}}$ for all $V_{G}$ and shifts the GIDL curve toward lower $V_{G}$ (again no skew; not shown). However, a skew (larger drop in $I_{\mathrm{GIDL}}$ at more negative $V_{G}$ ) in the GIDL curve was observed compared 


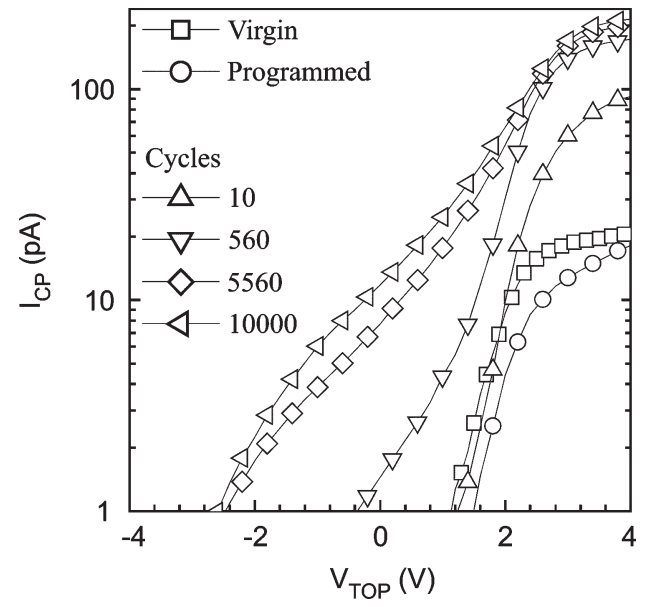

Fig. 14. $I_{\mathrm{CP}}$ measured in virgin state, after programming from virgin state, and in programmed state after different number of $\mathrm{P} / \mathrm{E}$ cycles. A gate pulse with a fixed base and varying top was used for measuring $I_{\mathrm{CP}}$.

to no-charge case when trapped charge in packet $\mathrm{A}$ and $\mathrm{B}$ differs in sign, with positive charge in packet $\mathrm{A}$ and negative charge in packet B. A similar skew was not observed with negative charge in packet $\mathrm{A}$ and positive charge in packet B. In addition, presence of interface traps was also found to decrease $I_{\mathrm{GIDL}}$ at more negative $V_{G}$ and resulted in a skew similar to experiments. The condition of positive charge in $\mathrm{A}$ and negative charge in $\mathrm{B}$ can occur in program-state if $\mathrm{P} / \mathrm{E}$ cycling results in accumulation of trapped holes in the overlap region (note that excess electrons can be stored above the channel region by performing program after $10 \mathrm{k}$ P/E cycles). But as erase-state GIDL characteristics also show a skew after cycling (Fig. 12), it is clear that formation of charge dipole is not the only reason for GIDL skew. Interface trap generation appears to be another (additional) likely cause behind this skew.

To verify the presence of positive charge in the overlap and/or interface traps in cycled cells, Fig. 14 shows virgin state $I_{\mathrm{CP}}$ and programmed state $I_{\mathrm{CP}}$ measured at different stages during cycling. The maximum $I_{\mathrm{CP}}$ continually increases with cycling and the rising edge of $I_{\mathrm{CP}}-V_{\mathrm{TOP}}$ curve gradually shifts to more negative $V_{\text {TOP }}$ with increasing P/E cycles. As mentioned earlier, hot-hole injection causes interface trap generation and the continual increase in $I_{\mathrm{CP}}$ shows that $N_{\mathrm{IT}}$ increases continuously with cycling. This makes interface trap generation one of the causes for reduction in GIDL and disturb with cycling. On the other hand, shift in the rising edge of $I_{\mathrm{CP}}-V_{\mathrm{TOP}}$ curve to more negative $V_{\mathrm{TOP}}$ values shows a reduction in the minimum local $V_{\mathrm{TH}}$ of the cell with cycling. As the local $V_{\mathrm{TH}}$ has minima in source/drain regions near the gate edges (in present conditions), this indicates a gradual increase of positive charge above the drain region with $\mathrm{P} / \mathrm{E}$ cycles. Thus, it is likely that reduction in disturb with P/E cycling occurs due to reduction in BTBT, which (in present conditions) is both due to positive charge accumulation above the overlap and interface degradation.

Simulations were used to further explore the influence of trapped charge and interface traps on BTBT under disturb condition. Trapped charge and interface traps were placed using a similar scheme as mentioned earlier. It was found that current arising from BTBT reduces in the presence of either positive

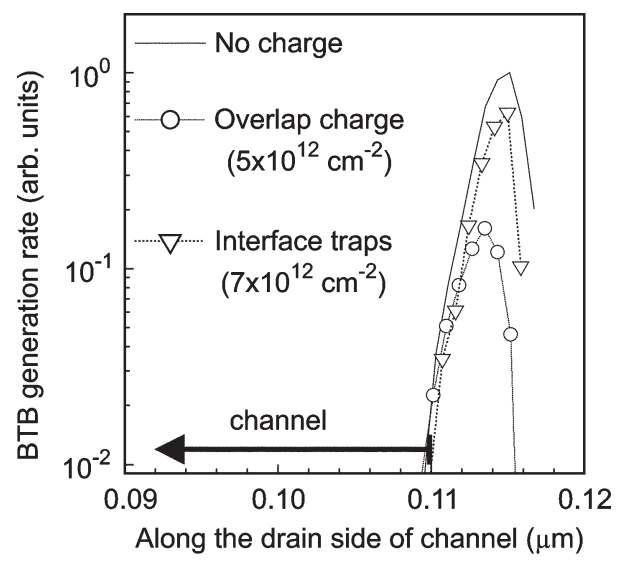

Fig. 15. Simulated band-to-band generation rate across the channel (near interface) of a SONOS cell under disturb condition, in the presence of ONO trapped charge and interface traps. The drain junction is at about $0.11 \mu \mathrm{m}$ and the center of channel is at origin. Trapped charge and interface traps are placed using a similar scheme as mentioned in Fig. 13. Band-to-band generation rate decreases in the presence of positive overlap charge (packet $\mathrm{A}$ ) or interface traps.

trapped charge in the overlap, or interface traps. Fig. 15 shows the simulated band-to-band generation rate across the channel (near interface) for two sample cases. The peak band-to-band generation rate decreases in presence of positive trapped charge or interface traps. This reduction in band-to-band generation rate is found to occur due to the increase of potential near the interface in drain region. Both positive overlap charge and interface traps, which also contribute positive charge when unoccupied (donor type), can increase this potential. While trap-assisted tunneling also can increase in the presence of interface traps, here this may be offset by the reduction in BTBT itself due to increased potential. Thus, both interface traps and positive charge trapping near the drain junction can reduce drain disturb.

\section{CONCLUSION}

Program drain disturb in SONOS Flash EEPROMs is investigated. Programmed SONOS cells suffer a severe charge loss disturb caused by injection of hot holes from substrate into the nitride. The two main sources of these holes are determined to be BTBT generation in the drain region, and channel leakage through impact ionization. BTBT is dominant in normal (no compensation) cells with and without halo doping for all $L$ and compensated cells at large $L$. Channel leakage becomes dominant in compensated cells as $L$ decreases and can cause severe bottleneck for cell scaling.

It is seen that program-state drain disturb becomes less severe with P/E cycling. The reasons behind this reduced disturb are analyzed using simulations. Simulations indicate that lower disturb is caused by reduction in BTBT generation, which in turn is either due to the presence of interface traps or accumulation of positive trapped charge in overlap region. Measurements show that $\mathrm{P} / \mathrm{E}$ cycling results in a continuous increase in interface traps and also leads to a gradual accumulation of positive charge near the overlap (under given conditions), establishing both of them to be the causes behind reduced disturb. The reduction 
in BTBT also affects erase speed during cycling and using a slightly longer erase time is needed to maintain a good cycling window.

\section{ACKNOWLEDGMENT}

The authors would like to thank D. R. Nair and J. Vasi of Indian Institute of Technology, Bombay, India, for useful discussions, J. Bude (formerly of Agere Systems, USA) for providing the Monte Carlo simulator, and S. Kamohara, E. Murakami, and K. Kubota (Renesas Technologies, Japan) for samples and support.

\section{REFERENCES}

[1] International Technology Roadmap for Semiconductors, 2005, San Jose, CA: Semiconductors Industry Association.

[2] B. Eitan, P. Pavan, I. Bloom, E. Aloni, A. Frommer, and D. Finzi, "Can NROM, a 2-bit, trapping storage NVM cell, give a real challenge to floating gate cells?" in Proc. SSDM, Sep. 1999, pp. 522-524.

[3] C. T. Swift, G. L. Chindalore, K. Harber, T. S. Harp, A. Hoefler, C. M. Hong, P. A. Ingersoll, C. B. Li, E. J. Prinz, and J. A. Yater, "An embedded $90 \mathrm{~nm}$ SONOS nonvolatile memory utilizing hot electron programming and uniform tunnel erase," in IEDM Tech. Dig., 2002, pp. 927-930.

[4] S. T. Kang, S. W. Park, Y. S. Park, B. Y. Seo, K. W. Kho, S. G. Kim, J. W. Han, S. B. Yoon, and C. H. Chung, "A study of SONOS nonvolatile memory cell controlled structurally by localizing charge-trapping layer," in Proc. NVSMW, 2003, pp. 39-41.

[5] Y. Yang and M. H. White, "Charge retention of scaled SONOS nonvolatile memory devices at elevated temperatures," Solid State Electron., vol. 44, no. 6, pp. 949-958, Jun. 2000

[6] W. J. Tsai, S. H. Gu, N. K. Zous, C. J. Liu, C. C. Liu, C. H. Chen, T. Wang, S. Pan, and C. Y. Lu, "Data retention behavior of a SONOS type two-bit storage Flash memory cell," in IEDM Tech. Dig., 2001, pp. 719-722.

[7] E. Lusky, Y. Shacham-Diamand, I. Bloom, and B. Eitan, "Electrons retention model for localized charge in oxide-nitride-oxide (ONO) dielectric," IEEE Electron Device Lett., vol. 23, no. 9, pp. 556-558, Sep. 2002.

[8] P. B. Kumar, R. Sharma, P. R. Nair, D. R. Nair, S. Kamohara, S. Mahapatra, and J. Vasi, "Mechanism of drain disturb in SONOS Flash EEPROMs," in Proc. IRPS, 2005, pp. 186-190.

[9] W. D. Brown and J. E. Brewer, Eds., Nonvolatile Semiconductor Technology. Piscataway, NJ: IEEE Press, 1998.

[10] D. R. Nair, S. Mahapatra, S. Shukuri, and J. D. Bude, "Drain disturb during CHISEL programming of NOR Flash EEPROMs-Physical mechanisms and impact of technological parameters," IEEE Trans. Electron Devices, vol. 51, no. 5, pp. 701-707, May 2004.

[11] P. B. Kumar, E. Murakami, S. Kamohara, and S. Mahapatra, "Endurance and retention characteristics of SONOS EEPROMs operated using BTBT induced hot hole erase," in Proc. IRPS, 2006, pp. 699-700.

[12] L. Larcher, P. Pavan, and B. Eitan, "On the physical mechanism of NROM memory erase," IEEE Trans. Electron Devices, vol. 51, no. 10, pp. 1593-1599, Oct. 2004.

[13] T. Y. Chan, J. Chen, P. K. Ko, and C. Hu, "The impact of gateinduced leakage current on MOSFET scaling," in IEDM Tech. Dig., 1987, pp. 718-721.

[14] Users Manual, ISE TCAD, 2002, Mountain View, CA: Synopsis, Inc.

[15] J. D. Bude, M. R. Pinto, and R. K. Smith, "Monte Carlo simulation of CHISEL Flash memory cell," IEEE Trans. Electron Devices, vol. 47, no. 10 , pp. $1873-1881$, Oct. 2000

[16] P. R. Nair, P. B. Kumar, R. Sharma, S. Kamohara, and S. Mahapatra, "A comprehensive trapped charge profiling technique for SONOS Flash EEPROMs," in IEDM Tech. Dig., 2004, pp. 403-406.

[17] G. Groeseneken, H. E. Maes, N. Beltran, and R. F. De Keersmaecker, "A reliable approach to charge pumping measurements in MOS-transistors," IEEE Trans. Electron Devices, vol. ED-31, no. 1, pp. 42-53, Jan. 1984.

[18] W. K. Chim, S. E. Leang, and D. S. H. Chan, "Extraction of metaloxide-semiconductor field-effect-transistor interface state and trapped charge spatial distributions using a physics-based algorithm," J. Appl. Phys., vol. 81, no. 4, pp. 1992-2001, Feb. 1997.

[19] S. K. Lai, "Interface trap generation in silicon dioxide when electrons are captured by trapped holes," J. Appl. Phys., vol. 54, no. 5, pp. 2540-2546, May 1983
[20] S. Ogawa and N. Shiono, "Interface-trap generation induced by hot hole injection at the $\mathrm{Si} / \mathrm{SiO}_{2}$ interface," Appl. Phys. Lett., vol. 61, no. 7 , pp. 807-809, Aug. 1992.

[21] D. Varghese, S. Mahapatra, and M. A. Alam, "Hole energy dependent interface trap generation in MOSFET Si $/ \mathrm{SiO}_{2}$ interface," IEEE Electron Device Lett., vol. 26, no. 8, pp. 572-574, Aug. 2005.

[22] E. Lusky, Y. Shacham-Diamand, G. Mitenberg, A. Shappir, I. Bloom, and B. Eitan, "Investigation of channel hot electron injection by localized charge-trapping nonvolatile memory devices," IEEE Trans. Electron Devices, vol. 51, no. 3, pp. 444-451, Mar. 2004.

[23] M. Janai, I. Bloom, G. Cohen, E. Lusky, A. Shappir, and B. Eitan, "Data retention reliability model of NROM nonvolatile memory devices," IEEE Trans. Device Mater. Rel., vol. 4, no. 3, pp. 404-415, Sep. 2004.

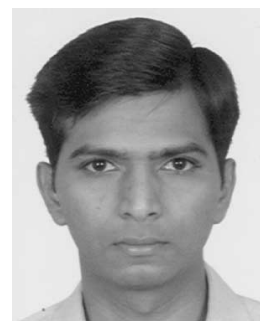

P. Bharath Kumar (S'04) received the M.Sc. degree in physics from Sri Sathya Sai Institute of Higher Learning, Prasanthinilayam, India, in 2001 and the M.Tech. degree in electrical engineering from the Indian Institute of Technology (IIT), Bombay, India, in 2003 , where he is currently working toward the Ph.D. degree.

His current research is focused on studying the reliability of Flash memory cells. Earlier he has worked on bias temperature instability in pMOS devices.

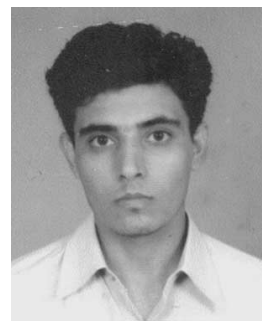

Ravinder Sharma received the M.Sc. degree in physics from Panjab University, Chandigarh, India, in 2002 and the M.Tech. degree in electrical engineering from the Indian Institute of Technology (IIT), Bombay, India, in 2004.

$\mathrm{He}$ is currently with STMicroelectronics Pvt. Ltd., Noida, India. His area of interest includes MOS transistors and Flash memories in devices, and system performance in design

Pradeep R. Nair received the B.Tech. degree in electronics and communication engineering from Regional Engineering College Calicut, India, in 2002 and the M.Tech. degree in electrical engineering from Indian Institute of Technology (IIT) Bombay, India, in 2004. He is currently working toward the Ph.D. degree in electrical engineering at Purdue University, West Lafayette, IN.

His current interests are characterization and numerical simulation of semiconductor devices and computational biophysics.

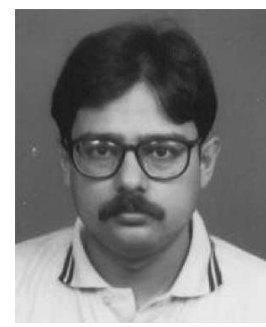

Souvik Mahapatra (S'99-M'99) received the Ph.D. degree in electrical engineering from the Indian Institute of Technology (IIT), Bombay, India, in 1999.

From 2000 to 2001, he was with Bell Laboratories, Lucent Technologies, Murray Hill, NJ. From 2002, he is with the Department of Electrical Engineering, IIT, where he is currently an Associate Professor. His research interests are electrical characterization of defects in dielectric-semiconductor interfaces, hotcarrier and bias temperature instability in CMOS devices, high- $\kappa$ and novel dielectrics for CMOS, and Flash electrically erasable programmable read-only memories. He has published more than 50 papers in refereed international journals and conferences.

Dr. Mahapatra was invited to speak at several major international conferences including the International Electron Devices Meeting (IEDM) and has worked as a reviewer for many international journals and conferences. 